\title{
Simultaneous isotope dilution quantification and metabolic tracing of deoxyribonucleotides by liquid chromatography high resolution mass spectrometry
}

\author{
Rostislav Kuskovsky ${ }^{1}$, Raquel Buj ${ }^{2}$, Peining $\mathrm{Xu}^{1}$, Samuel Hofbauer ${ }^{3}$, Mary T Doan ${ }^{1}$, Helen \\ Jiang $^{1}$, Anna Bostwick ${ }^{1}$, Clementina Mesaros ${ }^{3}$, Katherine M. Aird ${ }^{2}$, Nathaniel W. Snyder ${ }^{1,}$ \\ 1-AJ Drexel Autism Institute, Drexel University, 3020 Market St Suite 560, Philadelphia, PA 19104 \\ 2-Department of Cellular and Molecular Physiology, Penn State College of Medicine, Hershey, PA \\ 3-Centers for Cancer Pharmacology and Excellence in Environmental Toxicology, Department of \\ Pharmacology, University of Pennsylvania Perelman School of Medicine, Philadelphia, PA
}

\section{Abstract}

Quantification of cellular deoxyribonucleoside mono- (dNMP), di- (dNDP), triphosphates (dNTPs) and related nucleoside metabolites are difficult due to their physiochemical properties and widely varying abundance. Involvement of dNTP metabolism in cellular processes including senescence and pathophysiological processes including cancer and viral infection make dNTP metabolism an important bioanalytical target. We modified a previously developed ion pairing reversed phase chromatography- mass spectrometry method for the simultaneous quantification and ${ }^{13} \mathrm{C}$ isotope tracing of dNTP metabolites. dNMPs, dNDPs, and dNTPs were chromatographically resolved to avoid mis-annotation of in-source fragmentation. We used commercially available ${ }^{13} \mathrm{C}^{15} \mathrm{~N}$-stable isotope labeled analogs as internal standards and show that this isotope dilution approach improves analytical figures of merit. At sufficiently high mass resolution achievable on an Orbitrap mass analyzer, stable isotope resolved metabolomics allows simultaneous isotope dilution quantification and ${ }^{13} \mathrm{C}$ isotope tracing from major substrates including ${ }^{13} \mathrm{C}$-glucose. As a proof of principle, we quantified dNMP, dNDP and dNTP pools from multiple cell lines. We also identified isotopologue enrichment from glucose corresponding to ribose from the pentose-phosphate pathway in dNTP metabolites.

\section{Keywords}

dNTP; nucleotide; metabolism; high resolution mass spectrometry

\section{Introduction}

Intracellular deoxyribonucleoside triphosphate (dNTP) supply is tightly controlled by de novo synthesis, salvage, and degradation pathways $(1,2)$. Aberrant concentrations of dNTPs and their metabolites are associated with control of senescence $(3-5)$, cancer $(6,7)$,

*Corresponding author: Nws28@drexel.edu, AJ Drexel Autism Institute, Drexel University, 3020 Market St Suite 560, Philadelphia, PA 19104, Tel: 215-571-3492, Fax: 215.571.3187. 
metabolic diseases $(3,8)$, neurodegeneration (9), and viral infection (10). Basal dNTP levels during G1/G0 are extremely low (femtomolar range) and are mainly used for DNA repair and mitochondrial DNA synthesis $(11,12)$. During S phase, dNTP levels increase ten-fold to accommodate nuclear DNA replication (13). Excess dNTP levels reduce genome stability, replication fidelity, and reduce the length of, or delay entry into $S$ phase (14). On the other hand, low dNTP levels are deleterious by increasing the erroneous incorporation of rNMPs as well as the incidence of replication stress, leading to fork arrest, collapse, and double strand breaks (15). Mitochondrial depletion also results from the imbalance of dNTP levels (16). Therefore, dNTP pool balance is critical for the health of the cell.

Low basal concentrations of dNTPs and interference from analogs including NTPs, dideoxynucleoside triphosphates and mono- or di-phosphate deoxynucleosides presents an analytical challenge $(17,18)$. Detection with an enzymatic assay, in which a radioactive or fluorescence labeled dNTP is incorporated by a DNA polymerase proportionally to the unknown complementary base, is relatively sensitive with limits of detection (LoD) in the low pmol range (12). DNA polymerases sometimes incorporate rNTPs, thus often overestimating the concentration of dNTPs, especially at low dNTP concentrations or in complex matrices $(12,19)$. Furthermore, the multiplexing ability of these assays are limited and they cannot simultaneously quantify all nucleosides and their corresponding mono-, di-, and tri- phosphate forms (20). Liquid chromatography (LC)-UV detection methods are useful in dNTP bioanalysis but are limited in sensitivity, specificity, and multiplexing ability (21).

Mass spectrometry (MS) based quantification of dNTP metabolism offers unique benefits in terms of multiplexing, sensitivity and specificity. However, in-source fragmentation in electrospray ionization (ESI) sources of tri-phosphates to mono- and di-phosphates is problematic without chromatographic resolution or preparative separation of the mono-, diand tri-phosphates (22). Specifically, this complicates direct infusion high resolution mass spectrometry (HRMS) methods developed for other nucleotide metabolites (23). Triple quadrupole LC-tandem MS (LC-MS/MS) based methods have been developed to provide increased multiplexing and more specific measurements of dNMP and dNTP metabolites at adequate sensitivity for most biological samples (24). Methods for dNDPs are sparse in the literature, despite the biological importance of the generation of these metabolites via ribonucleotide reductase. Alternatively, due to the analytical challenges in quantifying dNTPs, many studies indirectly quantify dNTP metabolism by strategically assaying precursor metabolites including ribose-5-phosphate (R5P) (25). We aimed to leverage these benefits and overcome the remaining challenges in dNTP quantification by creating a method that could simultaneously perform quantification and isotope tracing on dNM/D/TPs within a single experiment and analytical run.

MS based methods can incorporate isotope dilution by adding an isotopically labeled internal standard at the beginning of a bioanalytical workflow, and then performing analysis on the ratio of the detected analyte/internal standard to adjust for matrix effects and losses during extraction and analysis (26). ${ }^{13} \mathrm{C},{ }^{15} \mathrm{~N}$-labeled ribonucleosides are available commercially, but methods to date have not utilized extensive isotope dilution. Aside from use of stable isotopes for more rigorous quantification, MS-based analysis can also enable 
isotope tracing, where the incorporation of a stable isotope labeled substrate in a metabolically active system is quantified by measuring the incorporation of the isotope label into an analyte. Synthesis of dNTPs incorporate carbon and nitrogen from diverse precursors, in separate pathways for purines and pyrimidines (Fig. 1). Carbon sources for de novo dNTP synthesis include glucose, aspartate, serine, and glycine whereas nitrogen is derived from glutamine, or aspartate (13). These precursors can be derived from multiple metabolic pathways, including glycolysis, glutaminolysis, and TCA cycle metabolism (3). Preferential utilization of these metabolites through each of these pathways is both cell-type and context dependent. Therefore, tracing the fate of atoms from different metabolites to $\mathrm{dNM} / \mathrm{D} / \mathrm{TP}$ pools is useful for understanding pathophysiological metabolic rewiring and pharmacological intervention.

To overcome the analytical challenges of dNTP quantification and maximize the analytical benefits of MS based analysis, we developed an ion pairing LC-HRMS method for the simultaneous isotope dilution based quantification and ${ }^{13} \mathrm{C}$-isotope tracing of dNTP from major carbon precursors. Although existing methods highlighted above have included quantification of dNMPs and dNTPs, and other methods have traced major carbon substrates into dNTP precursors, no method to date has combined isotope tracing and quantification by isotope dilution. Since this would be inherently difficult at mass resolution, we created a novel method using LC-HRMS and commercially available ${ }^{13} \mathrm{C},{ }^{15} \mathrm{~N}$-labeled dNMPs and dNTPs. Mono-, di-, and tri-phosphates of deoxyribonucleosides were quantified across different cell lines, and isotopic incorporation from ${ }^{13} \mathrm{C}$-glucose was examined. We demonstrate improved analytical parameters over label-free quantification and the ability to discern the contribution of major carbon sources into biologically informative patterns of isotope incorporation.

\section{Materials and Methods}

\section{Chemicals}

Standards for dAMP, ADP, dADP, ATP, dATP, dTMP, dTDP, dTTP, GMP, dGMP, GDP, dGDP, GTP, dGTP, CMP, dCMP, CDP, dCDP, CTP, dCTP, UMP, dUMP, UDP were from Sigma-Aldrich (St Louis, MO). Stable isotope labeled internal standards AMP- ${ }^{13} \mathrm{C}_{10},{ }^{15} \mathrm{~N}_{5}$, dAMP- ${ }^{13} \mathrm{C}_{10},{ }^{15} \mathrm{~N}_{5}$, ATP- $-{ }^{13} \mathrm{C}_{10},{ }^{15} \mathrm{~N}_{5}$, dATP- ${ }^{13} \mathrm{C}_{10},{ }^{15} \mathrm{~N}_{5}$, dTMP- ${ }^{13} \mathrm{C}_{10},{ }^{15} \mathrm{~N}_{2}$, dTTP- ${ }^{13} \mathrm{C}_{10},{ }^{15} \mathrm{~N}_{2}$, GMP- ${ }^{13} \mathrm{C}_{10},{ }^{15} \mathrm{~N}_{5}$, dGMP- ${ }^{13} \mathrm{C}_{10},{ }^{15} \mathrm{~N}_{5}$, GTP- ${ }^{13} \mathrm{C}_{10},{ }^{15} \mathrm{~N}_{5}$, dGTP- ${ }^{13} \mathrm{C}_{10},{ }^{15} \mathrm{~N}_{5}$, dCMP- ${ }^{13} \mathrm{C}_{9},{ }^{15} \mathrm{~N}_{3}$, CTP- ${ }^{13} \mathrm{C}_{9},{ }^{15} \mathrm{~N}_{3}$, dCTP- ${ }^{13} \mathrm{C}_{9},{ }^{15} \mathrm{~N}_{3}$, UTP- ${ }^{13} \mathrm{C}_{9},{ }^{15} \mathrm{~N}_{2}$, CMP- ${ }^{13} \mathrm{C}_{9},{ }^{15} \mathrm{~N}_{3}$, UMP- ${ }^{13} \mathrm{C}_{9},{ }^{15} \mathrm{~N}_{2}$ were also from Sigma-Aldrich. No suitable source of dUDP or stable isotope labeled ADP, dADP, dTDP, GDP, dGDP, CDP, dCDP, dUMP, UDP, dUDP, or dUTP was found.

Diisopropylethylamine (DIPEA) and 1,1,1,3,3,3-hexafluoro 2-propanol (HFIP), were purchased from Sigma-Aldrich. Optima LC-MS grade water, methanol, and acetonitrile (ACN) were purchased from Thermo Fisher Scientific (Waltham, MA). 


\section{Cell culture}

Normal, diploid IMR90 human fibroblasts were cultured according to the ATCC in low oxygen (2\%) in DMEM (4.5 g/L glucose, Corning cat\#10017CV) with 10\% FBS supplemented with L-glutamine, non-essential amino acids, sodium pyruvate, and sodium bicarbonate. Experiments were performed on IMR90 between population doubling \#25-35. Cells were routinely tested for mycoplasma as described in (27). Experiments targeting Ribonucleotide Reductase Regulatory Subunit M2 (RRM2), p16, mammalian target of rapamycin (mTOR), and ATR serine/threonine kinase (ATR) were previously included in Buj, et al.(28). Lentiviral constructs were transfected into 293FT cells using Lipofectamine 2000 (Thermo Fisher, Cat\# 11668019). Lentivirus was packaged using the ViraPower Kit (Invitrogen, cat\# K497500) following the manufacturer's instructions. Briefly, IMR90 were infected with pLKO.1 or pLKO.1-shRRM2 (Sigma-Aldrich, TRCN0000049410) and 24 hours later cells were infected with pLKO.1 or pLKO.1-shp16 (Sigma-Aldrich, TRCN0000010482). Cell were selected with puromycin $(3 \mu \mathrm{g} / \mathrm{mL})$ for seven days. Cells were treated at day 4 with $0.5 \mathrm{nM}$ of Temsirolimus an mTOR inhibitor (Selleckchem cat\#S1044) or VE822 an ATR inhibitor (cat\#S7102). After that, media was replaced for DMEM without glucose and glutamine (Sigma-Aldrich, cat\#D5030) and supplemented with 0.5\% of charcoal stripped FBS (Sigma-Aldrich, cat\#F6765-500ML), 20mM HEPES (Fisher BioReagents, cat\# BP310) and 5mM of $\left[{ }^{13} \mathrm{C}_{6}\right]$-D-glucose (Sigma-Aldrich, Cat\# 389374) or natural isotopic abundance D-glucose (Sigma-Aldrich, cat\#G8644). Labeling was stopped after 8 hours. Cells were washed twice with PBS and harvested with trypsin (Corning cat\#25-053-Cl). Pelleted cells were snap frozen in liquid nitrogen and stored at -80 Celsius. Cell pellets were extracted before analysis by addition of a $50 \mu \mathrm{L}(20 \mathrm{ng} / \mu \mathrm{L})$ mix of all stable isotope labeled internal standards (1000 ng/sample) in 80:20 (v/v) methanol:water followed by $1 \mathrm{~mL}$ of $-80^{\circ} \mathrm{C} 80: 20(\mathrm{v} / \mathrm{v})$ methanol:water.

\section{Liquid chromatography-high resolution mass spectrometry}

LC-HRMS was as previously described with minor modifications (29). Briefly, an Ultimate 3000 UHPLC equipped with a refrigerated autosampler (at $6{ }^{\circ} \mathrm{C}$ ) and a column heater (at 55 $\left.{ }^{\circ} \mathrm{C}\right)$ with a HSS C18 column $(2.1 \times 100 \mathrm{~mm}$ i.d., $3.5 \mu \mathrm{m}$; Waters, Milford, MA) was used for separations. Solvent A was $5 \mathrm{mM}$ DIPEA and $200 \mathrm{mM}$ HFIP and solvent B was methanol with $5 \mathrm{mM}$ DIPEA and $200 \mathrm{mM}$ HFIP. The gradient was as follows: $100 \%$ A for $3 \mathrm{~min}$ at $0.18 \mathrm{~mL} / \mathrm{min}, 100 \%$ A at $6 \mathrm{~min}$ with $0.2 \mathrm{~mL} / \mathrm{min}, 98 \%$ A at $8 \mathrm{~min}$ with $0.2 \mathrm{~mL} / \mathrm{min}, 86 \%$ A at $12 \mathrm{~min}$ with $0.2 \mathrm{~mL} / \mathrm{min}, 40 \% \mathrm{~A}$ at $16 \mathrm{~min}$ and $1 \% \mathrm{~A}$ at $17.9 \mathrm{~min}-18.5 \mathrm{~min}$ with 0.3 $\mathrm{mL} / \mathrm{min}$ then increased to $0.4 \mathrm{~mL} / \mathrm{min}$ until $20 \mathrm{~min}$. Flow was ramped down to $0.18 \mathrm{~mL} / \mathrm{min}$ back to $100 \%$ A over a 5 min re-equilibration. For MS analysis, the UHPLC was coupled to a Q Exactive HF mass spectrometer (Thermo Scientific, San Jose, CA, USA) equipped with a HESI II source operating in negative mode. The operating conditions were as follows: spray voltage $4000 \mathrm{~V}$; vaporizer temperature $200{ }^{\circ} \mathrm{C}$; capillary temperature $350{ }^{\circ} \mathrm{C}$; S-lens 60; in-source CID $1.0 \mathrm{eV}$, resolution 60,000. The sheath gas (nitrogen) and auxiliary gas (nitrogen) pressures were 45 and 10 (arbitrary units), respectively. Single ion monitoring (SIM) windows were acquired around the $[\mathrm{M}-\mathrm{H}]^{-}$of each analyte with a $20 \mathrm{~m} / \mathrm{z}$ isolation window, $4 \mathrm{~m} / z$ isolation window offset, $1 \mathrm{e}^{6}$ ACG target and $80 \mathrm{~ms}$ IT, alternating with a Full MS scan from 70-950 m/z with 1e6 ACG, and $100 \mathrm{~ms}$ IT. 


\section{Method Testing and Validation}

The resulting analytical method was tested for inter-day accuracy and precision and linearity. We estimated a lower limit of quantification (LLoQ) based on the lowest point of tested standard curves that fell within $25 \%$ of the expected value, since the signal intensity in the blank sample and the blank sample containing internal standard only had a signal intensity of 0 . We did not examine an upper limit of quantification since no sample values approached the upper end of our tested standard curve. Carryover was accessed by injection of a blank sample after each quality control level and was less than $0.1 \%$. Standard curves were prepared by serial dilution from $2000 \mathrm{ng} / \mathrm{sample}$, addition of a $50 \mu \mathrm{L}(20 \mathrm{ng} / \mu \mathrm{L}) \mathrm{mix}$ of all stable isotope labeled internal standards (1000 ng/sample) in 80:20 (v/v) methanol:water

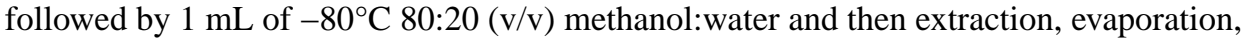
and resuspension identical to the samples. To test the properties of the method across levels

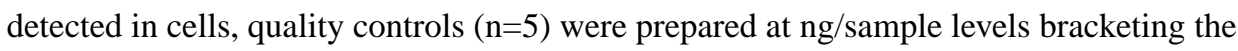
amounts detected in cells. This corresponded 3-levels for each analyte (ng/sample) were for $\operatorname{dTMP}(1,10,100)$, dCMP $(1,10,100)$, dADP $(100,200,600)$, dTDP $(10,100,600)$, dATP $(100,200,600)$. Coefficient of variation $(\% \mathrm{CV})$ was calculated from the (standard deviation/mean)*100. Accuracy (\%) was calculated as (observed-expected/expected)*100. The appropriate weighting method for interpolation of sample values was chosen based on the simplest method that resulted in quality control values $( \pm 20 \%)$ across the range found in samples.

\section{Statistical analysis}

Data was analyzed in XCalibur v4.0 and/or Tracefinder v4.1 (Thermo). Statistical analysis and graph generation was conducted in GraphPad Prism v7.04 (GraphPad Software La Jolla, CA).

\section{Results/Discussion}

\section{lon pairing reversed phase separation allows chromatographic resolution of intact mono-, di- and tri-phosphate forms of nucleosides}

Tri- and di-phosphates are known to easily lose a phosphate group in the ESI source to generate di- and mono-phosphates (22). Therefore, it is critical that the mono-, di-, and triphosphates of dNTP metabolites are resolved by chromatography. We first determined retention times and confirmed high abundance ions by injection of each individual standard. Mono-phosphates eluted in the 4-8 minute range, di- phosphates in 12-14.5 and triphosphates from 15- onwards. This provided baseline resolution of the major in-source fragments that had the same retention time of the precursor tri- or diphosphate. Standard for dAMP was cross-contaminated with other NTP metabolites and were not included in our analysis for purposes of absolute quantification. To confirm that this method was adequate for detection in cell samples, methanolic extract of IMR90 cells was analyzed and extracted ion chromatograms for all analytes and their available stable isotope labeled internal standards were plotted (Fig. S1). For analytes without matched isotope labeled analogs, the identity of peaks were confirmed by co-elution with pure standards to ensure misidentification with the in source fragment of related analytes (Fig. S2). Resolution of the insource fragments was maintained in cell matrix. Operation of the HRMS in full scan alone 
was insufficiently sensitive to detect all dNTP metabolites, thus in the final method single ion monitoring (SIM) was used, with a $20 \mathrm{~m} / z$ window with an offset of $4 \mathrm{~m} / \mathrm{z}$ around each $\mathrm{m} / \mathrm{z}$ corresponding to each analyte $[\mathrm{M}-\mathrm{H}]^{-}$. This window includes all internal standards used, and allowed for simultaneous mass isotopologue distribution analysis described below.

We were not able to chromatographically baseline resolve the isobaric dGTP/ATP, dGDP/ ADP, dGMP/AMP pairs in our system. Overlap of these nucleotides was noted in other methods, and negative mode fragmentation was reported to be less specific than positive ion mode. In the cases of both dGM/D/TPs, we could resolve the deoxynucleotides with LCMS/HRMS, but at drastically reduced sensitivity with more limited and complicated isotopologue analysis since the entire nucleotide is not captured by the detected ions (data not shown). Thus, we excluded this from analysis in this method. The concentration of AMP/ADP/ATP is expected to be orders of magnitude higher than the deoxyguanosine metabolites; thus, quantification by this method would reflect by majority AMP/ADP/ATP.

\section{Stable isotope dilution improves analytical performance of LC-HRMS based quantification of dNTPs}

Standard curves were generated for dNTPs except dAMP and dGM/D/TP. Pilot testing indicated that using higher amounts of dNTP internal standards improved detection in the lower range of standard curves, without interference from residual unlabeled impurities. Analysis of curves for dTM/D/P was conducted with isotope dilution or by label-free quantification using peak area around the levels detected in cell samples. For isotope dilution, the simplest model that fit the data with linear least-squares regression around the range detected in $1 \times 10^{6}$ cells $\left(0.97-500 \mathrm{ng} / \mathrm{sample}\right.$ ) was used, with $\mathrm{R}^{2}$ values of 0.9979 (no weighting), 0.9973 (1/x weighting), and 0.9933 (1/x weighting) for dTMP, dTDP, and dTTP, respectively. In comparison with the same weightings, label-free quantification gave $\mathrm{R}^{2}$ values of $0.9977,0.8258$, and 0.8592 (Fig. 2 A,B).

Calibration curves for $\mathrm{dCM} / \mathrm{D} / \mathrm{TP}$ were also linear across the range encountered in cell samples with excellent $\mathrm{R}^{2}$ values of $0.9999,0.9968,0.9965$ for dCMP, dCDP and dCTP respectively. Since previous methods have used surrogate internal standards (most commonly using 1 or two internal standards to normalize multiple analytes), we tested the utility of dCMP analysis by normalization to either the matched dCMP analog or dCTP. Normalization of dCMP signal area under the curve by dCTP-internal standard area under the curve yielded a poor least squares fit at the same concentration ranges (Fig. 2 C).

The signal intensity in the blank containing only isotope labeled internal standards had consistent background with 0 intensity. Therefore, for purposes of comparison to other methods of the limit of quantitation (LoQ), we conservatively estimated the LoQ as the first point that would fall within the experimentally confirmed linear range of the method with bias $<25 \%$ at $0.32 \mathrm{ng} / \mathrm{sample}$ for dTMP ( $49.9 \mathrm{fmol}$ on column), dTDP (39.7 fmol), dTTP (33.1 fmol), dCMP (52.0 fmol) dCDP (41.3 fmol), and dCTP (34.2 fmol). This was more than sufficient to quantify the levels within cells lines tested, and close to the previously reported LoQs of $62.5 \mathrm{fmol}$ for dNTPs (30). 
Quality controls were created to bracket levels observed in cell studies and from remnant tumor and non-tumor pathologic tissue (Table 1). This ranged from $1 \mathrm{ng} / \mathrm{sample}$ to $600 \mathrm{ng} /$ sample with pmol amounts reported for on column and pmol per sample for the lowest quality control.

To examine the effect of isotope dilution versus label free quantification we analyzed data from IMR90 cells with various short hairpin knockdown of genes found to disrupt nucleotide metabolism (28). This provided a bioanalytical meaningful way to modulate dNTP metabolites within similar cellular backgrounds. For this analysis, the analyst was blinded to identity of each sample (with respect to control versus knockdown and inhibition). Comparison of values obtained from quantification from cells revealed better precision with isotope dilution using analysis of the area under the curve (analyte/internal standard) than area under the curve of the analyte alone for dTMP (Fig. 3 A,D), dTDP (Fig. 3 B,E), and dTTP (Fig. 3 C,F). Bland-Altman plots showing the \% difference between the estimated value by each method and the averages of both approaches revealed an interesting concentration dependent bias in in all three analytes, with the lower values exhibiting a stronger negative bias in label-free quantification. This bias was especially pronounced in the IMR90 cell lines which had the three lowest values of all dTM/D/TP metabolites. Labelfree quantification under-estimated the pools in IMR90 by around 175\%, 90\%, and 50\% for dTMP, dTDP and dTTP as calculated with the \% difference between the value obtained from interpolation of the standard curves from the area under the curve (analyte/internal standard) and area under the curve of the analyte alone. The reasons for this differential bias across nucleotide amounts is unknown, but has implications for the reporting of nucleotide metabolites from metabolomics data where isotope dilution approaches should be given greater metrological weight.

\section{Stable isotope resolved metabolomics of dNTPs is possible at ultra-high resolution}

Carbon atoms are derived from glucose, $\mathrm{CO}_{2}$ /bicarbonate, formate, aspartate, and glycine, with nitrogen atoms coming from glutamine and aspartate. Since none of these are essential amino acids or nutrients they can be derived from the diverse sources of all of these precursors as well. These precursors can be traced via stable isotope labeling strategies which incorporate atoms from a labeled substrate into the metabolic product. Isotopic tracing requires higher sensitivity than quantification as isotopologue analysis requires, by definition, analysis of less abundant isotopologues. Furthermore, tracing with a stable isotope splits the signal intensity across isotopologues as they incorporate the isotopic label. Theoretically, at sufficient mass resolution, simultaneous quantification and isotope tracing can be accomplished with differential neutron encoded labels (e.g., ${ }^{13} \mathrm{C}$ vs. ${ }^{15} \mathrm{~N}$ ) due to the mass defect in the additional neutron in atoms with varying nuclei (31).

At sufficient mass resolution, stable isotopes of commonly used tracing atoms $\left({ }^{2} \mathrm{H},{ }^{13} \mathrm{C},{ }^{15} \mathrm{~N}\right.$, ${ }^{18} \mathrm{O}$, etc.) can be resolved from each other. Thereby, neutron-encoded information enables simultaneous metabolic tracing by stable isotope labeling with orthogonal stable isotope labeling used as an internal standard for isotope dilution based quantification (31). Since dNTPs and nucleoside metabolites incorporate $\mathrm{C}, \mathrm{H}, \mathrm{N}$, and $\mathrm{O}$ atoms, and commercially available ${ }^{13} \mathrm{C},{ }^{15} \mathrm{~N}$-labeled pure standards are available for isotope dilution, we examined the 
ability to simultaneously trace ${ }^{13} \mathrm{C}$-labeling via metabolic tracing with ${ }^{13} \mathrm{C}_{6}$-glucose with ${ }^{13} \mathrm{C},{ }^{15} \mathrm{~N}$ internal standards. This allows an experimental design where the origin of dNTP pools can be quantitatively examined, by quantifying the substrate-product relationship from labeled precursors, as well as the relative importance of de novo versus different salvage or uptake pathways. The limiting factor is resolution of the ${ }^{13} \mathrm{C}$ and ${ }^{15} \mathrm{~N}$ labels, so we modeled theoretical resolution at different resolving powers. With Orbitrap mass analyzers, resolution falls off with increasing $\mathrm{m} / \mathrm{z}$ (32), thus the most difficult to resolve isotopologues are the triphosphates with the highest possible number of labels. 240,000 resolving power was sufficient to baseline resolve the ${ }^{15} \mathrm{~N}_{1}$ labeled isotopologue from ${ }^{13} \mathrm{C}_{1}$ labels (Fig. $4 \mathrm{~A}-\mathrm{D}$ ). A lower resolution setting would be capable of resolving the dNMPs, or utilization of MS/ HRMS and analysis of the dNMP product ions (Fig. 4E).

We tested this theoretical possibility by a proof-of-principle experiment using isotope dilution absolute quantification in the analysis of a ${ }^{13} \mathrm{C}_{6}$-glucose labeling experiment. IMR 90 cells were grown with or without ${ }^{13} \mathrm{C}_{6}$-glucose, and with either empty vector control or RRM2 knockdown. We analyzed the dTM/D/TP pool size by isotope dilution based quantification (Fig. $5 \mathrm{~A}$ ) and the isotopologue enrichment of each analyte (Fig. 5 B,C,D). We integrated the peak area of each isotopologue of dTM/D/TP corresponding to the sequential incorporation of ${ }^{13} \mathrm{C}$ atoms. After correction for natural isotopic abundance isotopologue enrichment of dTM/D/TP revealed the quantitative incorporation of ${ }^{13} \mathrm{C}$ labeled glucose in a pattern of mixed sequential M1 and M2 labeling with a large increase in M5 labeling and some M6 labeling. This peak at M5 likely corresponds to the incorporation of ${ }^{13} \mathrm{C}_{5}$.

Since full scan data was acquired in addition to the targeted SIMs, we re-interrogated the data for relative abundance and labeling into ribose-5-phosphate, the pentose-phosphate pathway product that provides the ribose in dNTPs (Fig. 5 E,F). Labeling of ribose-5phosphate mirrored that of dNTPs, providing confirmation that we were measuring the incorporation of the ${ }^{13} \mathrm{C}$ into dNTPs. This indicates that in the lines and cell conditions tested, the contribution of glucose to dNTP synthesis is likely via the pentose phosphate pathway. Recent work has demonstrated the importance of nucleotide tracing to determine the context specific fate of substrates. For instance, that response of cancer cells to metformin correlates with nucleotide levels derived from TCA carbon sources but not the pentose phosphate pathway (33). Similarly, resistance to the antimetabolite gemcitabine correlates with glucose carbon flux specifically through the non-oxidative pentose phosphate pathway (34). Thus, tracing of metabolites to nucleotides/dNTPs may determine which patients will respond to metabolic inhibitors and reveal metabolic vulnerabilities for subsets of cancers. However, many studies have not confirmed tracing of metabolites from nucleotide precursors to dNTPs. It will be important in the future to further dissect the regulation of dNTP synthesis using tracing studies in both normal and diseased states.

There are two major caveats of this method. First, further improvement of this method could be made by chromatographically resolving the remaining isobars (AMP/dGMP, ADP/dGDP, ATP/dGTP). Although we could find a specific fragment on tandem MS/HRMS to differentiate the adenosine nucleotide phosphates from the deoxyguanosine nucleotide phosphates, this reduced sensitivity of the method below that useful for our purposes. While 
Olafsson, et al. were able to chromatographically separate dGTP and ATP, their method analyzed only the dNTPs (30). Other recent work has used a relatively simple derivatization resulting in methylation of the nucleotides, which allowed chromatographic resolution of AMP/dGMP, ADP/dGDP, and ATP/dGTP on a C18 stationary phase with a water/ acetonitrile gradient and detection by LC-MS/MS (35). Second, although we did not quantify it due to an impurity in the dAMP standard, sensitivity of dAMP was reduced by a high-intensity contaminant ion present across the LC-gradient falling in the same SIM window. This is problematic on our Orbitrap instrument as the sensitivity benefit gained from using the quadrupole for isolation to restrict ions allowed into the C-trap and Orbitrap is negated by a high-intensity background ion within the SIM window when the quadrupole isolation window was sufficient to allow complete isotopologue enrichment analysis. Further work to overcome these caveats will be necessary, including investigation alternative stationary phases, solvents, ion pairing reagents, or orthogonal methods of separation including ion mobility.

\section{Conclusion}

Direct quantification and isotope tracing of dNTP metabolites is analytically challenging. Ion pairing reversed phase LC coupled with Orbitrap based high-resolution mass spectrometry provides a platform for simultaneous isotope dilution based quantification of dNTPs with isotope tracing from multiple potential substrates.

\section{Supplementary Material}

Refer to Web version on PubMed Central for supplementary material.

\section{Acknowledgements}

This work was made possible by a NARSAD young investigator grant from the Brain and Behavior Foundation (NWS) as well as NIH grants K22ES026235 (NWS), R00CA194309 (KA), and P30ES013508.

\section{References}

1. Pai CC, Kearsey SE. A Critical Balance: dNTPs and the Maintenance of Genome Stability. Genes (Basel). 2017;8(2). Epub 2017/02/02. doi: 10.3390/genes8020057.

2. Hollenbaugh JA, Shelton J, Tao S, Amiralaei S, Liu P, Lu X, Goetze RW, Zhou L, Nettles JH, Schinazi RF, Kim B. Substrates and Inhibitors of SAMHD1. PLoS One. 2017;12(1):e0169052 Epub 2017/01/04. doi: 10.1371/journal.pone.0169052. [PubMed: 28046007]

3. Buj R, Aird KM. Deoxyribonucleotide Triphosphate Metabolism in Cancer and Metabolic Disease. Front Endocrinol (Lausanne). 2018;9:177 Epub 2018/05/04. doi: 10.3389/fendo.2018.00177. [PubMed: 29720963]

4. Santinon G, Brian I, Pocaterra A, Romani P, Franzolin E, Rampazzo C, Bicciato S, Dupont S. dNTP metabolism links mechanical cues and YAP/TAZ to cell growth and oncogene-induced senescence. EMBO J. 2018;37(11). Epub 2018/04/14. doi: 10.15252/embj.201797780.

5. Aird KM, Worth AJ, Snyder NW, Lee JV, Sivanand S, Liu Q, Blair IA, Wellen KE, Zhang R. ATM couples replication stress and metabolic reprogramming during cellular senescence. Cell Rep. 2015;11(6):893-901. Epub 2015/05/06. doi: 10.1016/j.celrep.2015.04.014. [PubMed: 25937285]

6. Kohnken R, Kodigepalli KM, Wu L. Regulation of deoxynucleotide metabolism in cancer: novel mechanisms and therapeutic implications. Mol Cancer. 2015;14:176 Epub 2015/09/30. doi: 10.1186/s12943-015-0446-6. [PubMed: 26416562] 
7. Aird KM, Zhang G, Li H, Tu Z, Bitler BG, Garipov A, Wu H, Wei Z, Wagner SN, Herlyn M, Zhang R. Suppression of nucleotide metabolism underlies the establishment and maintenance of oncogeneinduced senescence. Cell Rep. 2013;3(4):1252-65. Epub 2013/04/09. doi: 10.1016/ j.celrep.2013.03.004. [PubMed: 23562156]

8. Shimizu I, Yoshida Y, Suda M, Minamino T. DNA damage response and metabolic disease. Cell metabolism. 2014;20(6):967-77. Epub 2014/12/03. doi: 10.1016/j.cmet.2014.10.008. [PubMed: 25456739]

9. Fasullo M, Endres L. Nucleotide salvage deficiencies, DNA damage and neurodegeneration. Int J Mol Sci. 2015;16(5):9431-49. Epub 2015/04/30. doi: 10.3390/ijms16059431. [PubMed: 25923076]

10. Gizzi AS, Grove TL, Arnold JJ, Jose J, Jangra RK, Garforth SJ, Du Q, Cahill SM, Dulyaninova NG, Love JD, Chandran K, Bresnick AR, Cameron CE, Almo SC. A naturally occurring antiviral ribonucleotide encoded by the human genome. Nature. 2018 Epub 2018/06/22. doi: 10.1038/ s41586-018-0238-4.

11. Chen X, McAllister KJ, Klein B, Bushman LR, Anderson PL. Development and validation of an LC-MS/MS quantitative method for endogenous deoxynucleoside triphosphates in cellular lysate. Biomed Chromatogr. 2017;31(3). Epub 2016/08/25. doi: 10.1002/bmc.3820.

12. Ferraro P, Franzolin E, Pontarin G, Reichard P, Bianchi V. Quantitation of cellular deoxynucleoside triphosphates. Nucleic Acids Res. 2010;38(6):e85 Epub 2009/12/17. doi: 10.1093/nar/gkp1141. [PubMed: 20008099]

13. Lane AN, Fan TW. Regulation of mammalian nucleotide metabolism and biosynthesis. Nucleic Acids Res. 2015;43(4):2466-85. Epub 2015/01/30. doi: 10.1093/nar/gkv047. [PubMed: 25628363]

14. Weinberg G, Ullman B, Martin DW Jr. Mutator phenotypes in mammalian cell mutants with distinct biochemical defects and abnormal deoxyribonucleoside triphosphate pools. Proceedings of the National Academy of Sciences of the United States of America. 1981;78(4):2447-51. [PubMed: 7017732]

15. Yao NY, Schroeder JW, Yurieva O, Simmons LA, O’Donnell ME. Cost of rNTP/dNTP pool imbalance at the replication fork. Proceedings of the National Academy of Sciences of the United States of America. 2013;110(32):12942-7. Epub 2013/07/25. doi: 10.1073/pnas.1309506110. [PubMed: 23882084]

16. Copeland WC. Inherited mitochondrial diseases of DNA replication. Annual review of medicine. 2008;59:131-46. Epub 2007/09/26. doi: 10.1146/annurev.med.59.053006.104646.

17. Hennere G, Becher F, Pruvost A, Goujard C, Grassi J, Benech H. Liquid chromatography-tandem mass spectrometry assays for intracellular deoxyribonucleotide triphosphate competitors of nucleoside antiretrovirals. Journal of chromatography B, Analytical technologies in the biomedical and life sciences. 2003;789(2):273-81. [PubMed: 12742119]

18. Cohen S, Megherbi M, Jordheim LP, Lefebvre I, Perigaud C, Dumontet C, Guitton J. Simultaneous analysis of eight nucleoside triphosphates in cell lines by liquid chromatography coupled with tandem mass spectrometry. Journal of chromatography B, Analytical technologies in the biomedical and life sciences. 2009;877(30):3831-40. Epub 2009/10/07. doi: 10.1016/ j.jchromb.2009.09.030. [PubMed: 19805008]

19. Wilson PM, Labonte MJ, Russell J, Louie S, Ghobrial AA, Ladner RD. A novel fluorescencebased assay for the rapid detection and quantification of cellular deoxyribonucleoside triphosphates. Nucleic Acids Res. 2011;39(17):e112 Epub 2011/05/18. doi: 10.1093/nar/gkr350. [PubMed: 21576234]

20. Roy B, Beuneu C, Roux P, Buc H, Lemaire G, Lepoivre M. Simultaneous determination of pyrimidine or purine deoxyribonucleoside triphosphates using a polymerase assay. Analytical biochemistry. 1999;269(2):403-9. Epub 1999/05/01. doi: 10.1006/abio.1999.4051. [PubMed: 10222017]

21. Decosterd LA, Cottin E, Chen X, Lejeune F, Mirimanoff RO, Biollaz J, Coucke PA. Simultaneous determination of deoxyribonucleoside in the presence of ribonucleoside triphosphates in human carcinoma cells by high-performance liquid chromatography. Analytical biochemistry. 1999;270(1):59-68. Epub 1999/05/18. doi: 10.1006/abio.1999.4066. [PubMed: 10328765] 
22. Xu YF, Lu W, Rabinowitz JD. Avoiding misannotation of in-source fragmentation products as cellular metabolites in liquid chromatography-mass spectrometry-based metabolomics. Anal Chem. 2015;87(4):2273-81. Epub 2015/01/17. doi: 10.1021/ac504118y. [PubMed: 25591916]

23. Lorkiewicz P, Higashi RM, Lane AN, Fan TW. High information throughput analysis of nucleotides and their isotopically enriched isotopologues by direct-infusion FTICR-MS. Metabolomics. 2012;8(5):930-9. Epub 2012/10/27. doi: 10.1007/s11306-011-0388-y. [PubMed: 23101002]

24. Chen P, Liu Z, Liu S, Xie Z, Aimiuwu J, Pang J, Klisovic R, Blum W, Grever MR, Marcucci G, Chan KK. A LC-MS/MS method for the analysis of intracellular nucleoside triphosphate levels. Pharm Res. 2009;26(6):1504-15. Epub 2009/03/18. doi: 10.1007/s11095-009-9863-9. [PubMed: 19291372]

25. Ben-Sahra I, Howell JJ, Asara JM, Manning BD. Stimulation of de novo pyrimidine synthesis by growth signaling through mTOR and S6K1. Science. 2013;339(6125):1323-8. Epub 2013/02/23. doi: 10.1126/science.1228792. [PubMed: 23429703]

26. Ciccimaro E, Blair IA. Stable-isotope dilution LC-MS for quantitative biomarker analysis. Bioanalysis. 2010;2(2):311-41. Epub 2010/03/31. doi: 10.4155/bio.09.185. [PubMed: 20352077]

27. Uphoff CC, Drexler HG. Detection of mycoplasma contaminations. Methods Mol Biol. 2005;290:13-23. [PubMed: 15361652]

28. Buj R, Kuskovsky R, Dahl ES, Leon KE, Maglakelidze N, Navaratnarajah M, Zhang G, Doan MT, Jiang H, Kutzler L, Lacko H, Lu Y, Mills GB, Gowda R, Robertson GP, Herlyn M, Imamura Y, Kimball S, Snyder NW, Aird KM. Suppression of p16 increases nucleotide synthesis via mTORC1. bioRxiv. 2018. doi: 10.1101/393876.

29. Guo L, Worth AJ, Mesaros C, Snyder NW, Glickson JD, Blair IA. Diisopropylethylamine/ hexafluoroisopropanol-mediated ion-pairing ultra-high-performance liquid chromatography/mass spectrometry for phosphate and carboxylate metabolite analysis: utility for studying cellular metabolism. Rapid Commun Mass Spectrom. 2016;30(16):1835-45. Epub 2016/08/02. doi: 10.1002/rcm.7667. [PubMed: 27476658]

30. Olafsson S, Whittington D, Murray J, Regnier M, Moussavi-Harami F. Fast and sensitive HPLCMS/MS method for direct quantification of intracellular deoxyribonucleoside triphosphates from tissue and cells. Journal of chromatography B, Analytical technologies in the biomedical and life sciences. 2017;1068-1069:90-7. Epub 2017/10/17. doi: 10.1016/j.jchromb.2017.10.008.

31. Frey AJ, Feldman DR, Trefely S, Worth AJ, Basu SS, Snyder NW. LC-quadrupole/Orbitrap highresolution mass spectrometry enables stable isotope-resolved simultaneous quantification and (1) (3)C-isotopic labeling of acyl-coenzyme A thioesters. Anal Bioanal Chem. 2016;408(13):3651-8. Epub 2016/03/13. doi: 10.1007/s00216-016-9448-5. [PubMed: 26968563]

32. Makarov A, Denisov E, Lange O. Performance evaluation of a high-field Orbitrap mass analyzer. J Am Soc Mass Spectrom. 2009;20(8):1391-6. Epub 2009/02/14. doi: 10.1016/j.jasms.2009.01.005. [PubMed: 19216090]

33. Liu X, Romero IL, Litchfield LM, Lengyel E, Locasale JW. Metformin Targets Central Carbon Metabolism and Reveals Mitochondrial Requirements in Human Cancers. Cell metabolism. 2016;24(5):728-39. Epub 2016/10/18. doi: 10.1016/j.cmet.2016.09.005. [PubMed: 27746051]

34. Shukla SK, Purohit V, Mehla K, Gunda V, Chaika NV, Vernucci E, King RJ, Abrego J, Goode GD, Dasgupta A, Illies AL, Gebregiworgis T, Dai B, Augustine JJ, Murthy D, Attri KS, Mashadova O, Grandgenett PM, Powers R, Ly QP, Lazenby AJ, Grem JL, Yu F, Mates JM, Asara JM, Kim JW, Hankins JH, Weekes C, Hollingsworth MA, Serkova NJ, Sasson AR, Fleming JB, Oliveto JM, Lyssiotis CA, Cantley LC, Berim L, Singh PK. MUC1 and HIF-1alpha Signaling Crosstalk Induces Anabolic Glucose Metabolism to Impart Gemcitabine Resistance to Pancreatic Cancer. Cancer Cell. 2017;32(1):71-87 e7 Epub 2017/07/12. doi: 10.1016/j.ccell.2017.06.004. [PubMed: 28697344]

35. Li Z, Zhang HX, Li Y, Lam CWK, Wang CY, Zhang WJ, Wong VKW, Pang SS, Yao MC, Zhang W. Method for Quantification of Ribonucleotides and Deoxyribonucleotides in Human Cells Using (Trimethylsilyl)diazomethane Derivatization Followed by Liquid Chromatography-Tandem Mass Spectrometry. Anal Chem. 2018 Epub 2018/12/12. doi: 10.1021/acs.analchem.8b04281. 


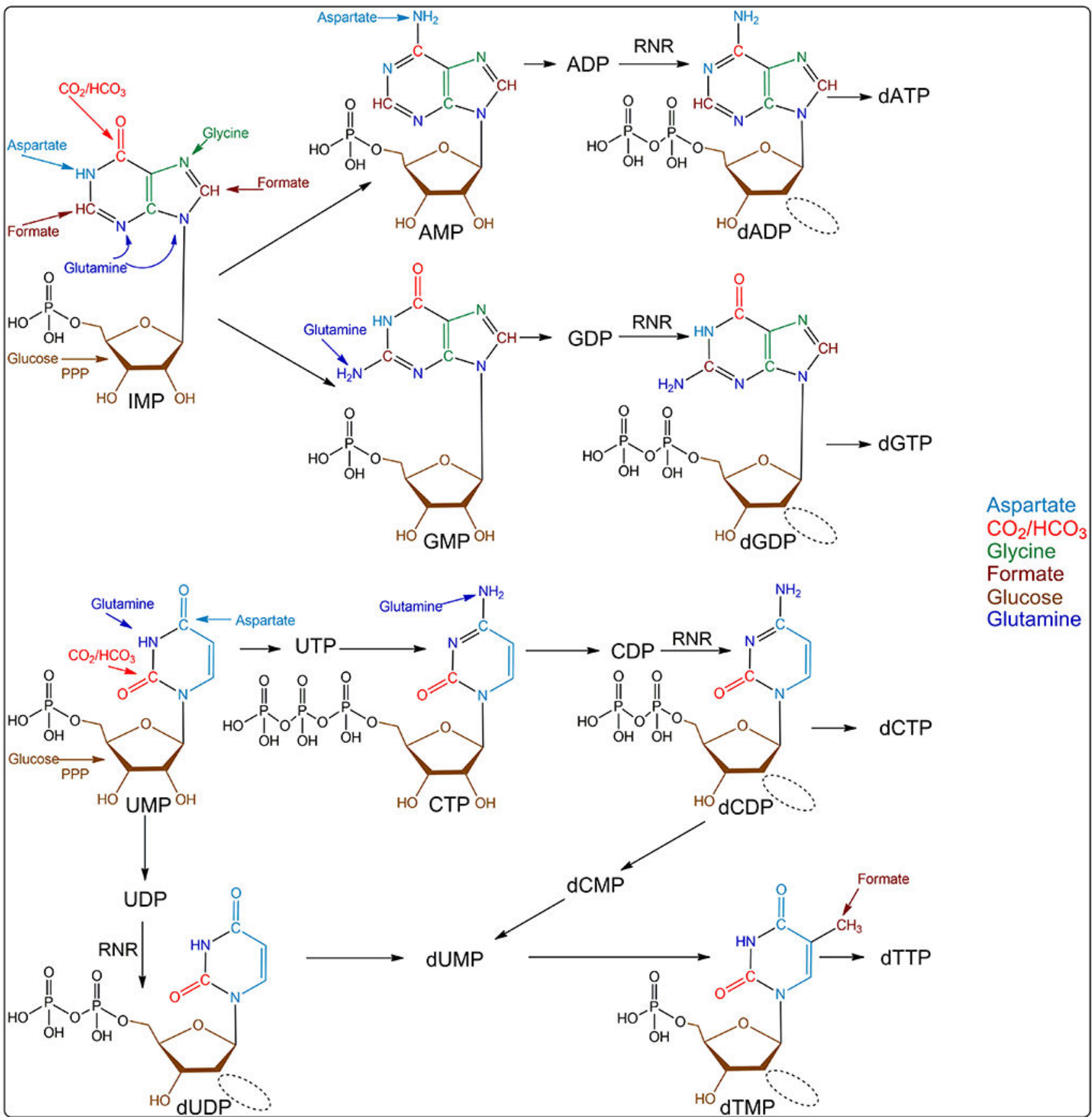

Figure 1.

dNTP metabolism integrates atoms from a number of potential substrates. 

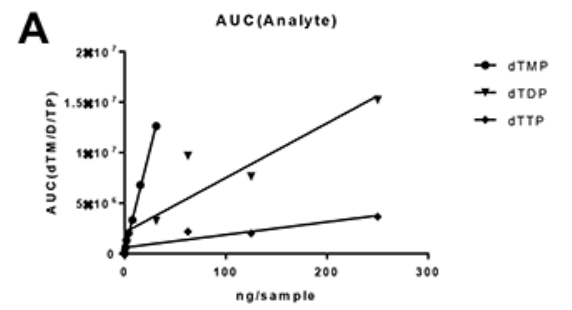

B

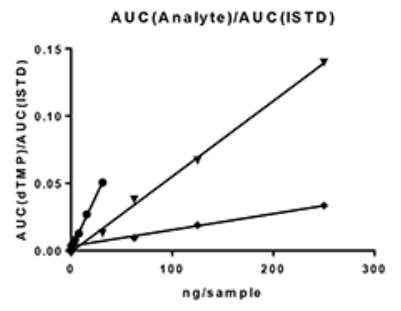

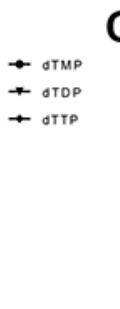

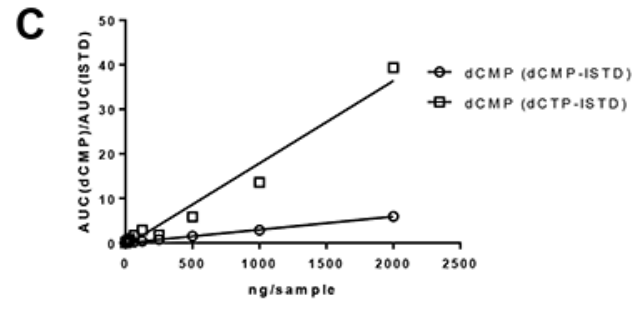

Figure 2.

Calibration curves of dTM/D/P in cell samples using A) label-free quantification B) isotope dilution. C) Calibration curves of dCMP using dCMP-internal standard (ISTD) or dCTPISTD. 
A

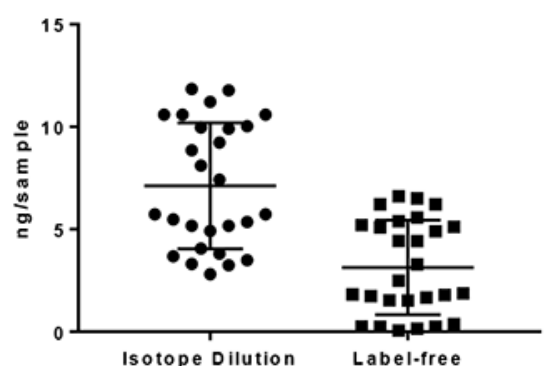

D

\% Difference vs, average: dTMP

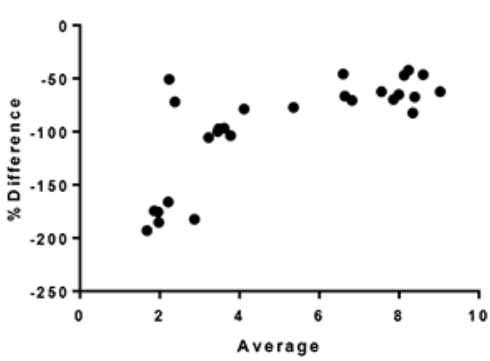

B

d T D P

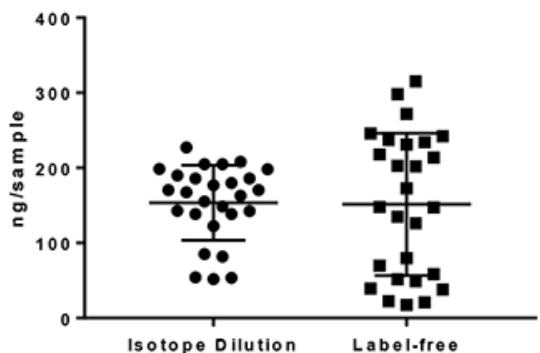

$\mathbf{E}$

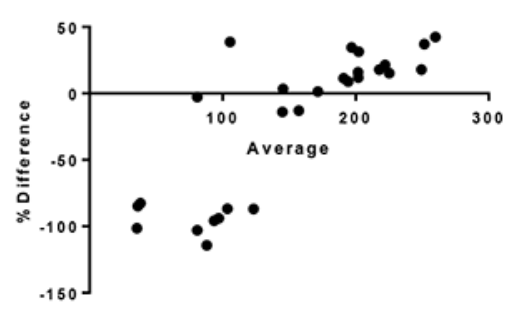

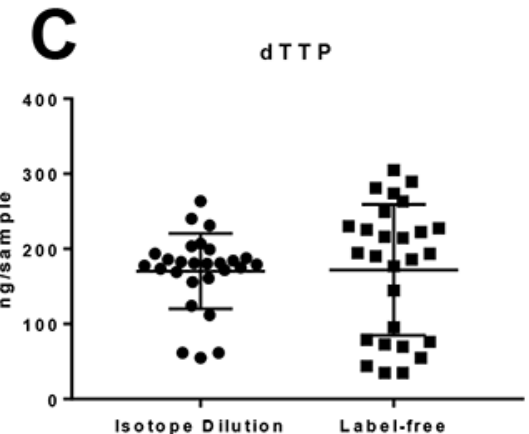

$\mathbf{F}$

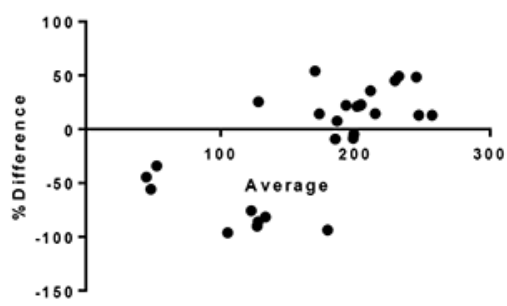

Figure 3.

Bias in quantification of dTM/D/TP in cell samples using isotope dilution or label-free quantification. Quantification of A) dTMP B) dTDP C) dTTP. Bland-Altman plot showing $\%$ difference in isotope dilution versus label-free quantification for D) dTMP E) dTDP and F) dTTP. 

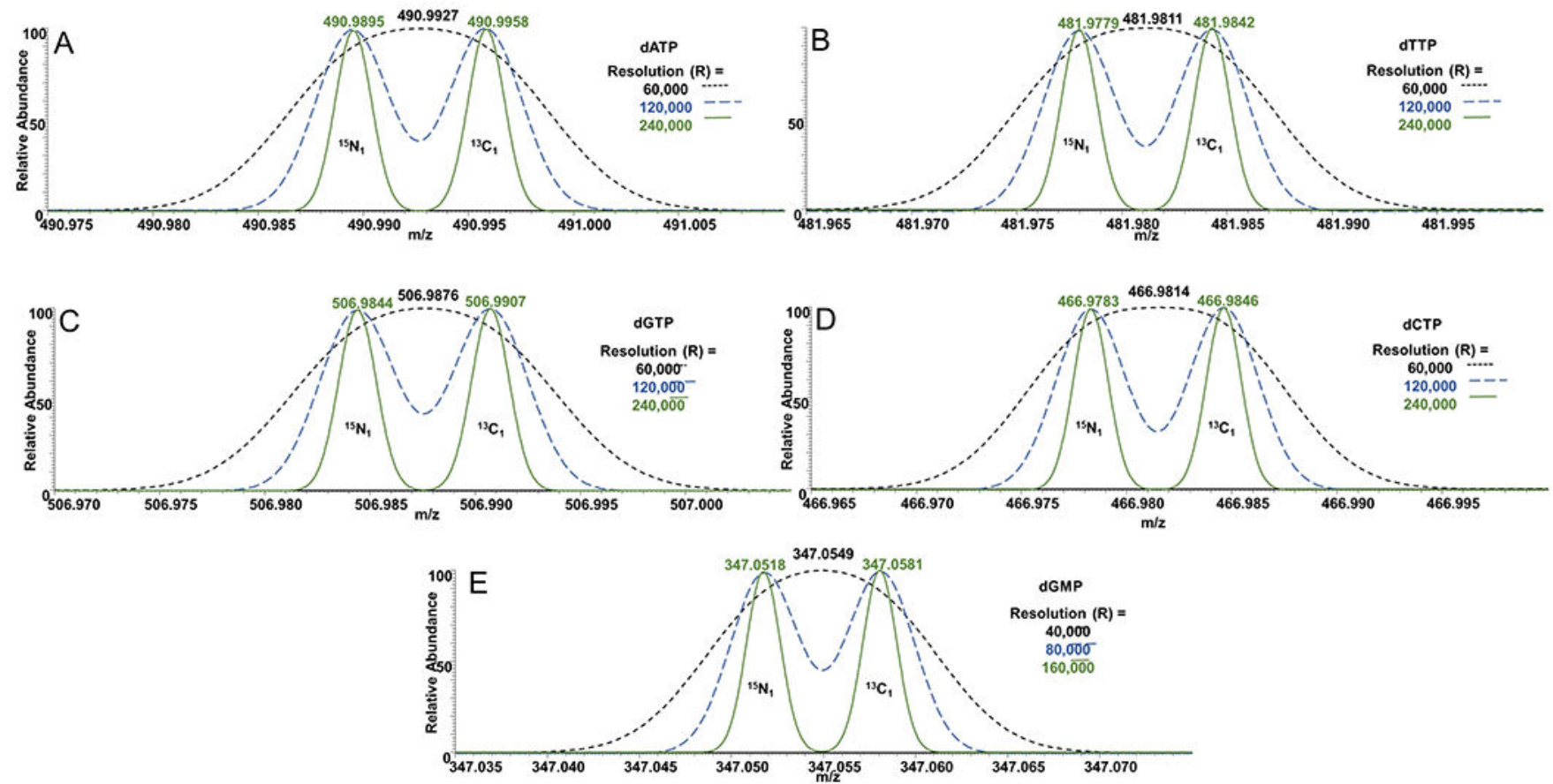

Figure 4.

Resolution of the dNTPs and their ${ }^{13} \mathrm{C} /{ }^{15} \mathrm{~N}$ isotopologues requires ultra-high resolution mass spectrometry. Theoretical Gaussian resolution (FWHM) with 40 samples per peak is shown at increasing levels of resolving power per nucleotide. Predicted centroid masses for $[\mathrm{M}-\mathrm{H}]^{-}$ at each resolution are shown for a 1:1 mixture each of (A) ${ }^{13} \mathrm{C}_{1} \mathrm{dATP} /{ }^{15} \mathrm{~N}_{1} \mathrm{dATP}$ (B) ${ }^{13} \mathrm{C}_{1} \mathrm{dTTP} /{ }^{15} \mathrm{~N}_{1} \mathrm{dTTP}(\mathrm{C}){ }^{13} \mathrm{C}_{1} \mathrm{dGTP} /{ }^{15} \mathrm{~N}_{1} \mathrm{dGTP}$ (D) ${ }^{13} \mathrm{C}_{1} \mathrm{dCTP} /{ }^{15} \mathrm{~N}_{1} \mathrm{dCTP}$ and (E) ${ }^{13} \mathrm{C}_{1} \mathrm{dGMP} /{ }^{15} \mathrm{~N}_{1} \mathrm{dGMP}$. 

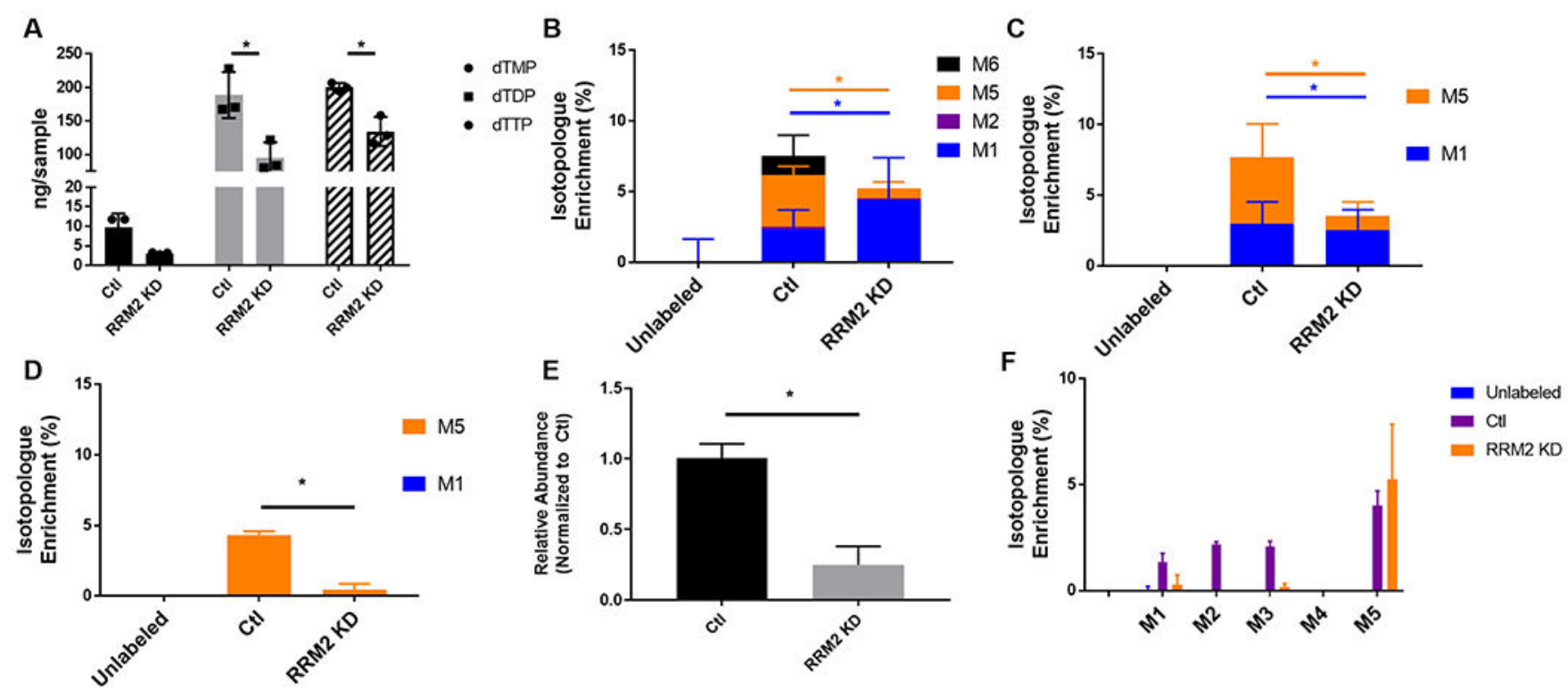

Figure 5.

Simultaneous isotope dilution based quantification and isotope tracing of dTM/D/P in ${ }^{13} \mathrm{C}$ labeled IMR90 extract. A) Quantification of dTM/D/TP, isotopologue enrichment (\%) of B) dTMP C) dTDP and D) dTTP. Full scan data was integrated for E) relative quantification of ribose-5-phosphate (R5P) F) isotopologue enrichment (\%) of R5P. Where indicated, * $\mathrm{p}<$ 0.05 . 
Table 1.

Analytic figures of merit for quality control samples bracketing cell and tissue levels of dNM/D/TPs.

\begin{tabular}{|c|c|c|c|c|c|c|c|c|c|c|c|c|}
\hline & & & & $\begin{array}{c}1 \mathrm{ng} / \\
\text { sample }\end{array}$ & & $\begin{array}{l}10 \mathrm{ng} / \\
\text { sample }\end{array}$ & & $\begin{array}{l}100 \mathrm{ng} / \\
\text { sample }\end{array}$ & & $\begin{array}{l}200 \mathrm{ng} / \\
\text { sample }\end{array}$ & & $\begin{array}{l}600 \mathrm{ng} / \\
\text { sample }\end{array}$ \\
\hline Analyte & M.W. & $\begin{array}{l}\text { Internal } \\
\text { Standard }\end{array}$ & Model & $\begin{array}{c}\text { Precision } \\
(\% \mathrm{CV})\end{array}$ & $\begin{array}{c}\text { AccuracY } \\
\text { (\% Bias) }\end{array}$ & $\begin{array}{c}\text { Precision } \\
\text { (\% CV })\end{array}$ & $\begin{array}{l}\text { Accuracy } \\
\text { (\% Bias) }\end{array}$ & $\begin{array}{c}\text { Precision } \\
(\% \mathrm{CV})\end{array}$ & $\begin{array}{c}\text { Accuracy } \\
\text { (\% Bias) }\end{array}$ & $\begin{array}{c}\text { Precision } \\
(\% \mathrm{C} \text { V })\end{array}$ & $\begin{array}{c}\text { Accuracy } \\
\text { (\%8ias) }\end{array}$ & $\begin{array}{c}\text { Precision } \\
\text { (\% CV })\end{array}$ \\
\hline dAMP & 331.22 & & N/A & & & & & & & & & \\
\hline dADP & 411.20 & dATP & $\begin{array}{c}\text { no } \\
\text { weighting }\end{array}$ & & & & & $8 \%$ & $19 \%$ & $9 \%$ & $-6 \%$ & $9 \%$ \\
\hline dATP & 491.18 & dATP & $\begin{array}{c}\text { no } \\
\text { weighting }\end{array}$ & & & & & $5 \%$ & $18 \%$ & $8 \%$ & $-12 \%$ & $6 \%$ \\
\hline dTMP & 321.20 & dTTP & $\begin{array}{c}\text { no } \\
\text { weighting }\end{array}$ & $14 \%$ & $7 \%$ & $5 \%$ & $4 \%$ & $5 \%$ & $0 \%$ & & & \\
\hline dTDP & 402.19 & dTTP & $\begin{array}{c}1 / x \\
\text { weighting }\end{array}$ & & & $13 \%$ & $-18 \%$ & $6 \%$ & $3 \%$ & & & $23 \%$ \\
\hline dTTP & 482.17 & dTTP & $\begin{array}{c}1 / x \\
\text { weighting }\end{array}$ & & & $9 \%$ & $7 \%$ & $5 \%$ & $-2 \%$ & & & $2 \%$ \\
\hline $\mathrm{dCMP}$ & 307.20 & dCMP & $\begin{array}{c}\text { no } \\
\text { weighting }\end{array}$ & $22 \%$ & $-8 \%$ & $9 \%$ & $1 \%$ & $6 \%$ & $2 \%$ & & & $6 \%$ \\
\hline $\mathrm{dCOP}$ & 387.18 & $\mathrm{dCTP}$ & $\begin{array}{c}\text { no } \\
\text { weighting }\end{array}$ & & & & & $3 \%$ & $8 \%$ & & & $17 \%$ \\
\hline $\mathrm{dCTP}$ & 467.16 & $\mathrm{dCTP}$ & $\begin{array}{c}\text { no } \\
\text { weighting }\end{array}$ & & & & & $10 \%$ & $-3 \%$ & & & $13 \%$ \\
\hline
\end{tabular}

